\title{
An Efficient Adaptive Technique with Low Complexity for Reducing PAPR in OFDM-Based Cognitive Radio
}

\author{
Hefdhallah Sakran, ${ }^{1}$ Omar Nasr, ${ }^{2}$ and Mona Shokair ${ }^{1}$ \\ ${ }^{1}$ Faculty of Electronic Engineering, El-Menoufia University, Menouf, Egypt \\ ${ }^{2}$ Faculty of Engineering, Cairo University, Giza, Egypt \\ Correspondence should be addressed to Hefdhallah Sakran, hefdh_sakran@yahoo.com
}

Received 6 March 2012; Accepted 17 May 2012

Academic Editors: G. Camps-Valls and H. Hu

Copyright (C) 2012 Hefdhallah Sakran et al. This is an open access article distributed under the Creative Commons Attribution License, which permits unrestricted use, distribution, and reproduction in any medium, provided the original work is properly cited.

\begin{abstract}
Cognitive radio (CR) is considered nowadays as a strong candidate solution for the spectrum scarcity problem. On standards level, many cognitive radio standards have chosen Non-Contiguous Orthogonal Frequency Division Multiplexing (NC-OFDM) as their modulation scheme. Similar to OFDM, NC-OFDM suffers from the problem of having a high Peak to Average Power Ratio (PAPR). If not solved, either the transmitted signal will be distorted, which will cause interference to primary (licensed) users, or the effeciency of the power amplifier will be seriously degraded. The effect of the PAPR problem in NC-OFDM based cognitive radio networks is worse than normal OFDM systems. In this paper, we propose enhanced techniques to reduce the PAPR in NCOFDM systems. We start by showing that combining two standard PAPR reduction techniques (interleaver-based and selective mapping) results in a lower PAPR than using them individually. Then, an "adaptive number of interleavers" will be proposed that achieves the same performance of conventional interleaver-based PAPR reduction while reducing the CPU time by $41.3 \%$. Finally, adaptive joint interleaver with selective mapping is presented, and we show that it gives the same performance as conventional interleaver-based technique, with reduction in CPU time by a factor of $50.1 \%$.
\end{abstract}

\section{Introduction}

There is an unparalleled increase in the usage of wireless devices in the last decade. However, most of the frequency spectrum has already been licensed exclusively to operators by government agencies, such as Federal Communications Commission (FCC). Therefore, there exists an apparent spectrum scarcity for new wireless applications and services. In recent studies, especially by the FCC, it is reported that there are vast temporal and spatial variations in the allocated spectrum utilization. The spectrum utilization efficiency can be as low as $15 \%$ [1]. Recently, cognitive radio [2, 3] has attracted much attention, as it can solve the spectrum scarcity problem [4] by allowing cognitive users (unlicensed users) to occupy the spectrum band when the primary users (licensed users) do not use their licensed spectrum. The main functions that a cognitive radio can perform are: spectrum sensing, spectrum management, spectrum mobility, and Spectrum Sharing [4]. From standards point of view, most of the cognitive radio-based standards have selected OFDM, or a variation of it, as their modulation scheme. The challenges of OFDM-based cognitive radio can be grouped into three categories, which are described in details in [5]. One of these categories is related to PAPR problem. This problem has been thoroughly studied for OFDM systems. However, for Noncontiguous OFDM, which is used in cognitive radio networks, the problem is severer than normal OFDM systems. The main reason is that, in NC-OFDM systems, some subcarriers are used for primary users and the others are used for the secondary users. Any nonlinear effect due to the nonlinearity power amplifier for the secondary users' signal will cause leakage and Intercarrier Interference (ICI), which will disturb the primary user transmission.

Although many techniques were introduced to reduce PAPR problem in OFDM systems, these reduction algorithms need to be carefully chosen and modified to be used for NCOFDM systems [6]. The problem of reducing PAPR occurring in cognitive radio has been studied before [6-10]. 
A statistical analysis of PAPR for NC-OFDM system was presented in [6]. The authors in [7] proposed a joint sidelobe and PAPR reduction method that can simultaneously reduce the inherent OFDM out-of-band (OOB) radiation and the OOB radiation caused by a nonlinear power amplifier. In this method, selective mapping (SLM) sequences, which are generated from sequences with a low average sidelobe power, are used to improve the PAPR performance. The authors in [8] proposed Cognitive Noncontinuous Carrier Interferometry Orthogonal Signal Division Multiplexing (Cognitive NCI-OSDM) compared to NC-OFDM where Carrier Interferometry (CI) codes made a system with limited PAPR of the transmitted signal. In [9], the authors proposed an algorithm that exploits the agility of cognitive radio technology to rapidly choose and employ the appropriate PAPR reduction approach from a set of approaches to achieve a large decrease in PAPR. SLM is another technique to reduce PAPR in cognitive radio [10].

In this paper, we present three proposed techniques for reducing PAPR in NC-OFDM-based systems. We start by combining two-existing techniques (the interleaver-based reduction and the SLM), then we introduce two adaptive techniques to reach the same results as static techniques, while reducing the processing power tremendously.

The rest of this paper is organized as follows. In Section 2, PAPR for NC-OFDM system is introduced. In Section 3, proposed techniques will be investigated. Simulation results will be explained in Section 4. Finally, conclusions are made in Section 5.

\section{PAPR for NC-OFDM System}

The complex envelope of a baseband NC-OFDM signal, consisting of all $N$ contiguous subcarriers over a time interval $[0, T]$, is given by

$$
x(t)=\sum_{k=0}^{N-1} X_{k} e^{j 2 \pi k f_{0} t},
$$

where $f_{0}=1 / T, X_{k}$ is the symbol of the $k$ th subcarrier, and $T$ is the OFDM symbol duration. The symbol over the $k$ th deactivated subcarrier is $X_{k}=0$.

The PAPR of the signal, $x(t)$, is then given as the ratio of the peak instantaneous power to the average power and written as

$$
\operatorname{PAPR}=\max _{0 \leq t \leq T} \frac{|x(t)|^{2}}{E\left[|x(t)|^{2}\right]},
$$

where $E[\cdot]$ is the expectation operator.

\section{Proposed Techniques}

In NC-OFDM systems, distortion-based techniques, such as clipping and filtering, and frequency-domain-based techniques assuming contiguous subcarriers, such as coding, cannot be used for reducing PAPR [6]. However, frequencydomain PAPR reduction techniques such as interleaving,
SLM, and Partial Transmit Sequences (PTS) [11] are better suited, since it is easier to sort out the nulled subcarriers avoiding any interference to primary user. In the following, proposed techniques for reducing PAPR in NC-OFDM will be explained.

3.1. Joint Interleaver-SLM Reduction. The main idea of the first proposed technique is to use a combination of two frequency-domain PAPR reduction techniques that can be used for reducing PAPR of NC-OFDM signals. Interleavers and SLM have been used individually for NC-OFDM systems PAPR reduction. They are used together in our proposed technique. Figure 1 illustrates the block diagram of NCOFDM employing the proposed PAPR reduction algorithm.

In this approach, $M$ interleavers are used at the transmitter. These interleavers produce $M$ permuted frames of the input data sequence. The PAPR of all frames is estimated, and the minimum PAPR frame of all of them is then passed through SLM.

In the SLM technique, several different OFDM signals are generated by multiplying the original data block with $D$ different phase sequences, $B^{(d)}=\left[b_{d, 0}, b_{d, 1}, \ldots, b_{d, N-1}\right]^{T}$, and $d=1,2, \ldots, D$ to make the $D$ phase rotated OFDM data blocks, where the $D$ phase rotated OFDM data blocks is represented by the same information as the original OFDM data block. Then, from $D$ modified data blocks, the OFDM data block with minimum PAPR for transmission will be selected.

3.2. Adaptive Number of Interleavers. This scheme targets a lower power implementation of interleavers-based PAPR reduction techniques. It is mainly suitable for DSP-based implementations, where, unlike FPGA implementations, instructions run in a fetch-decode-execute cycle. The algorithm steps are explained as follows.

(1) PAPR of the symbol $X$ is compared to a threshold $\operatorname{PAPR}_{\mathrm{Th}}$. If $\operatorname{PAPR}\left(\sum_{k=0}^{N-1} X_{k} e^{j 2 \pi k f_{0} t}\right)<\operatorname{PAPR}_{\mathrm{Th}}, X$ is transmitted without any modification. The algorithm exists in this case.

(2) Set interleaver number to $1(i=1)$.

(3) Calculate $\operatorname{PAPR}_{i}=\operatorname{PAPR}\left(\sum_{k=0}^{N-1} X_{k}^{\prime} e^{j 2 \pi k f_{0} t}\right)$, where $X^{\prime}=\left[X_{\Pi_{i}(0)}, X_{\Pi_{i}(1)}, \ldots, X_{\Pi_{i}\left(N_{a}\right)}\right]^{T}$, and $\Pi(n) \in$ $\left\{0,1, \ldots, N_{a}-1\right\}$ is the interleaving map.

(4) If $\mathrm{PAPR}_{i}<\mathrm{PAPR}_{\mathrm{Th}}$, or all interleavers have been tested, then Exit.

(5) Select the next interleaver in the interleavers bank $(i=i+1)$.

(6) Go to step (2).

The flow chart in Figure 2 describes the steps of the proposed algorithm. The reduction in power comes from setting a predetermined PAPR $\mathrm{P}_{\mathrm{Th}}$ threshold and comparing the PAPR of the output of a certain interleaver to this threshold. For most of the packets, the algorithm will exit before going through all interleavers. This threshold depends on the system requirements. For example, in case of short-distance 


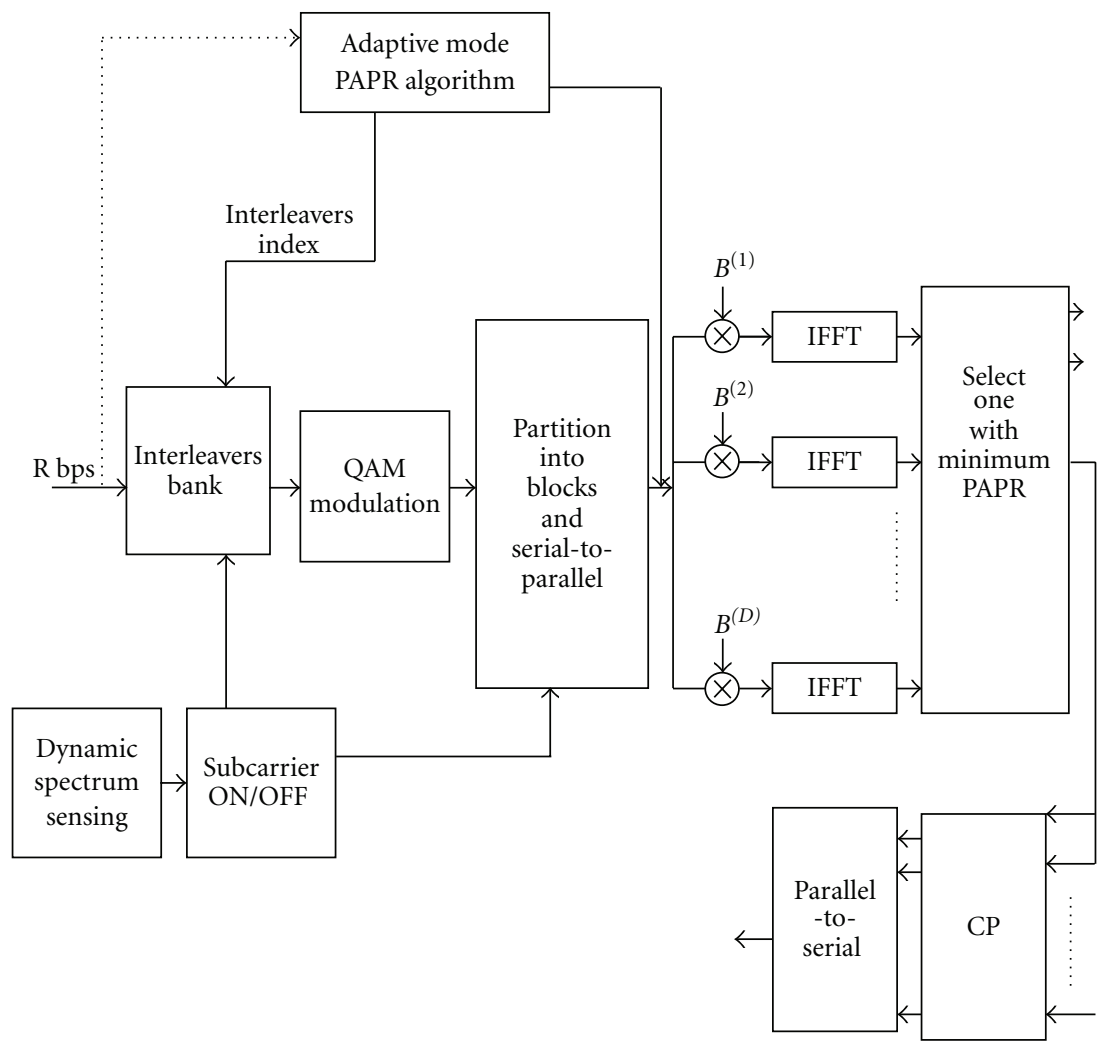

FIGURE 1: NC-OFDM system with proposed technique for PAPR reduction.

communications, the processing power is the dominant factor of the total-system power. However, in long distances, the total power of the power amplifier is the dominant factor. Hence, $\mathrm{PAPR}_{\mathrm{Th}}$ can be small in case of long distances and vice versa. This is similar to the treatment in [12], where the transceiver parameters change depending on the transmission distance.

3.3. Adaptive Number of Interleavers and Adaptive SLM. This algorithm combines the previous scheme (adaptive number of interleavers) with an adaptive SLM PAPR reduction scheme. A maximum of $M$ interleavers and D SLM can be used for any symbol. The algorithm works as follows.

(1) Apply the adaptive number of interleavers algorithm on the input symbol with half number of interleavers bank in the adaptive number of interleaver algorithm.

(2) If the PAPR resulting from step (1) is less than $\mathrm{PAPR}_{\mathrm{Th}}$, then Exit.

(3) Let $X_{n}^{\prime}$ is the permuted data block with lowest PAPR in step (1), set phase sequence number to $1(d=1)$.

(4) Calculate $\operatorname{PAPR}_{d}=\operatorname{PAPR}\left(\sum_{k=0}^{N-1} X_{k}^{d} e^{j 2 \pi k f_{0} t}\right)$, where $X_{k}^{d}=X_{k}^{\prime} e^{j \phi_{k}^{d}}, \phi_{k}^{d} \in[0, \pi]$.

(5) If PAPR $_{d} \leq \mathrm{PAPR}_{\mathrm{Th}}$, or all $D$ different sequences have been tested, then Exit. If all $D$ different sequences have been tested $(d=D)$ select the sequence $d^{\prime}=$ $\arg _{1<d<D} \min \operatorname{PAPR}\left[S_{k}^{d}\right], S_{k}^{d}=\sum_{k=0}^{N-1} X_{k}^{\prime} e^{j \phi_{k}^{d}} e^{j 2 \pi k f_{0} t}$.
(6) Select the next sequence in the total $D$ different sequences that used to reduce PAPR $(d=d+1)$.

(7) Go to step (3).

\section{Simulation Results}

We perform a Monte Carlo simulation to show the performance gains of the proposed algorithms. 10,000 different NC-OFDM symbols run through independent trials to obtain the average results per experiment. The simulated system uses an NC-OFDM signal with random location distributions of 16 active subcarriers. The total number of subcarriers tested are $N=64$ and $N=256$ using both QPSK and 16QAM.

PAPR statistics are given in terms of the Complementary Cumulative Distribution Function (CCDF). The CCDF shows the probability of having the PAPR of an NC-OFDM frame exceeding a given $\mathrm{PAPR}_{0}$

$$
\operatorname{CCDF}(\operatorname{PAPR}(x))=\operatorname{Pr}\left(\operatorname{PAPR}(x)>\operatorname{PAPR}_{0}\right) .
$$

Figure 3 shows the CCDF of PAPR for conventional OFDM system (with all subcarriers being active), and NC-OFDM using 16QAM. In Figure 3, $S_{a} / S_{t}$ is the ratio of the number of active subcarriers over the total number of subcarriers. It can be seen from Figure 3 that the sparser the OFDM symbol, the worse the PAPR properties of it. All plots are for 16 active subcarriers. The PAPR of the 16 subcarriers OFDM symbol is better than those of NC-OFDM with the 


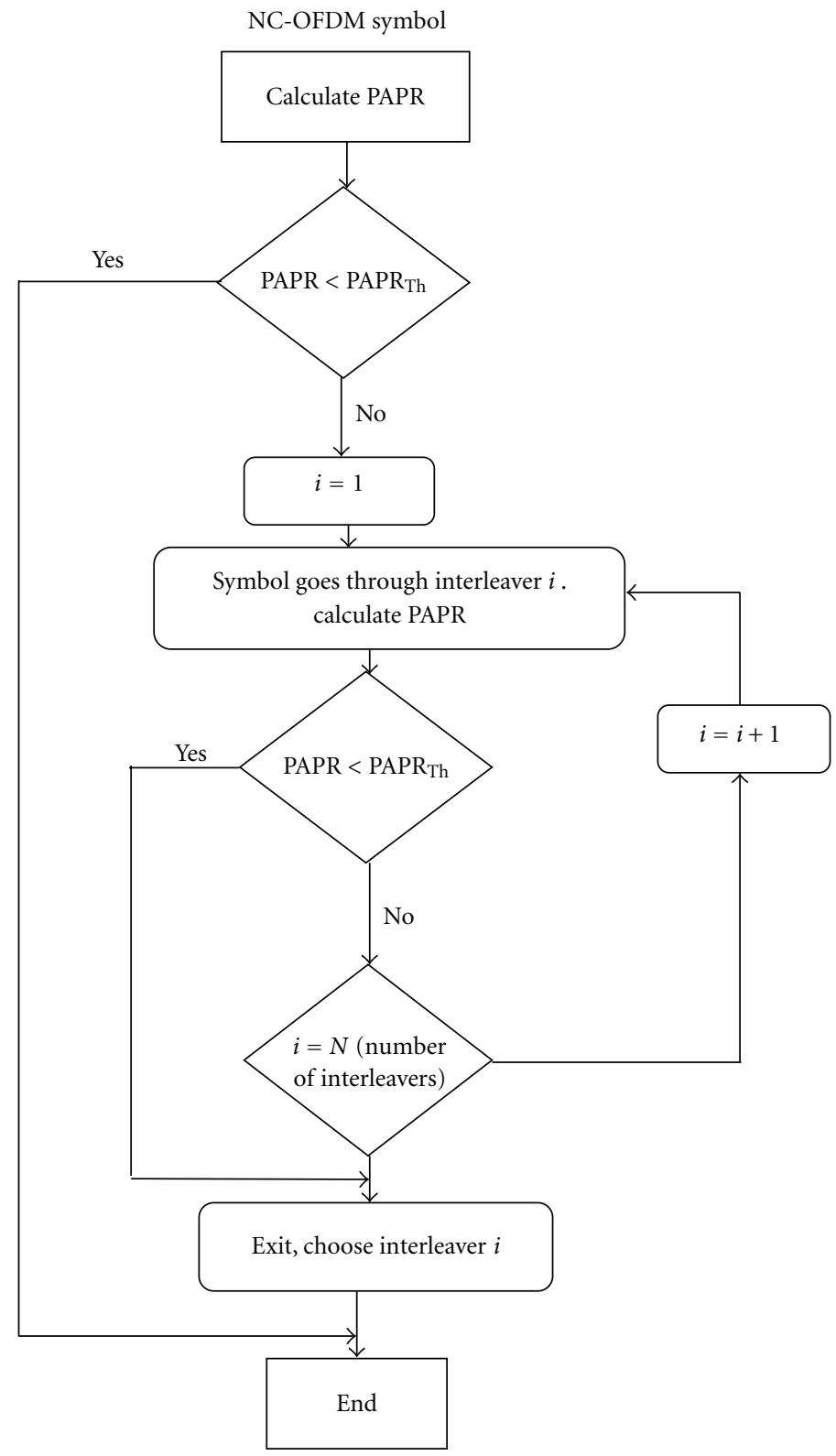

FIGURE 2: Adaptive interleaver algorithm.

same number of active subcarriers. Therefore, the problem of PAPR minimization is more challenging in NC-OFDM systems compared to conventional OFDM systems.

Figures 4(a) and 4(b) show the CCDF of PAPR for NCOFDM with $M=8$ interleaver and $D=4$ SLM sequences to reduce the PAPR using QPSK and 16QAM modulations, respectively. The figures also show the results in case of using the joint interleaver-SLM reduction technique. With the proposed technique, peak power at $\mathrm{CCDF}=10^{-3}$ reduces by $3.0 \mathrm{~dB}$ and $3.3 \mathrm{~dB}$ compared to the original system (NCOFDM without PAPR technique) for $16 / 256$ case and $16 / 64$ case, respectively. The reason for the PAPR improved value in $16 / 256$ case is less than that in $16 / 64$ case, because the ratio of the number of active subcarriers over the total number of subcarriers for $16 / 256$ case is smaller than in case $16 / 64$ and the proposed technique is more effective as the active subcarriers is large with respect to the total subcarriers.

Figure 5 compares the proposed adaptive number of interleavers algorithm with the traditional interleaver technique to reduce PAPR of NC-OFDM. It can be seen from this figure that the proposed algorithm gives the same performance of traditional interleaver technique. The proposed algorithm reduces the CPU time by $41.3 \%$ compared with the traditional interleaver technique. In this figure, we put PAPR $_{\text {Th }}=5.5$, where this value gives the same performance of traditional interleaver with $M=8$, and the maximum number of interleavers $=8$ for the proposed algorithm.

Figure 6 shows the CCDF performance of the proposed adaptive technique at different value of $\mathrm{PAPR}_{\mathrm{Th}}$. It is clear from this figure that the PAPR of NC-OFDM with proposed 


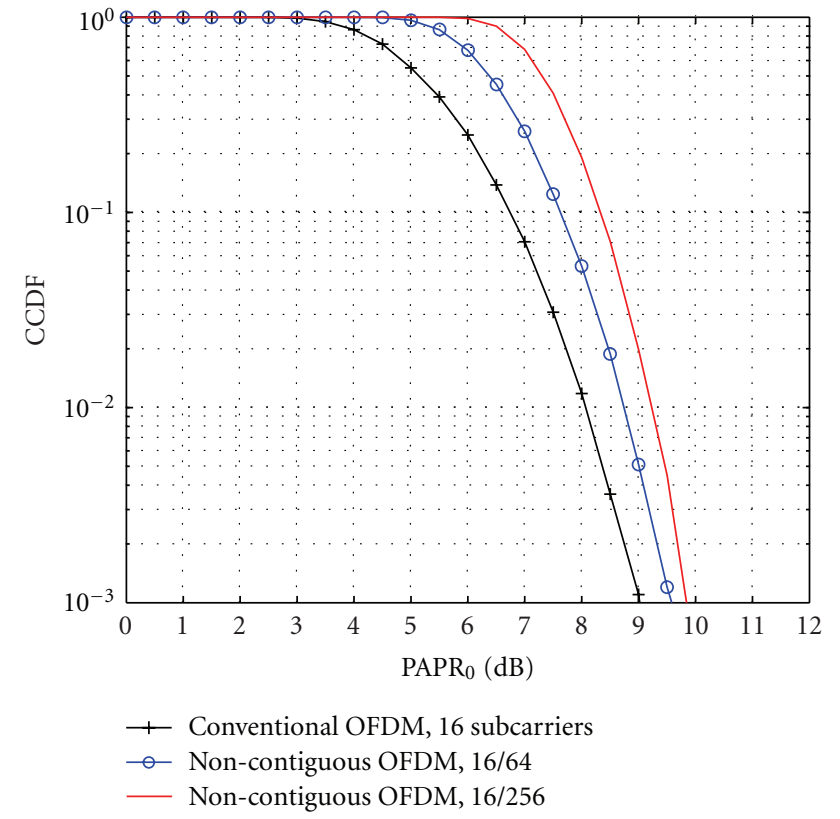

FIGURE 3: CCDF of PAPR for NC-OFDM compared with conventional OFDM.

adaptive technique is improved when the PAPR $\mathrm{P}_{\mathrm{Th}}$ values are decreased. It is also clear, that in the case of $\mathrm{PAPR}_{\mathrm{Th}}=5.5$, there are some frames still have PAPR larger than $\mathrm{PAPR}_{0}=$ 5.5 due to the proposed algorithm with maximum number of interleavers $(M=8)$ cannot reduce the PAPR less than 5.5, but in the case of $\mathrm{PAPR}_{\mathrm{Th}}=7$ the proposed algorithm is sufficient to reduce the PAPR less than PAPR $\mathrm{Th}_{\mathrm{Th}}$. It is also clear that, in the case of PAPR $\mathrm{Th}_{\mathrm{Th}}=7$, the CCDF is not identical to CCDF of NC-OFDM without PAPR reduction technique till the value of $\mathrm{PAPR}_{0}=7$, because the frames that have PAPR are larger than 7 , the interleaver is applied and the new value of PAPR for these frame is equal to any value from 0 to 7 . The interpretation in the case of $\mathrm{PAPR}_{\mathrm{Th}}=8$ is the same.

Figure 7 shows the average number of active interleavers with different values of $\mathrm{PAPR}_{\mathrm{Th}}$ in the adaptive number of interleavers algorithm. The average number of active interleavers is proportional to the average processing power in case of a DSP-based implementation. The lower the value of PAPR $\mathrm{Th}_{\mathrm{Th}}$, the higher the average number of interleaves used and the higher the processing power required in the PAPR reduction part.

To compare the processing power required for the traditional interleaver, the joint interleaver-SLM and the adaptive number of interleavers and adaptive SLM performances are plotted in Figure 8. The parameters for each algorithm were chosen to give the same performance as shown in Figure 8. Although the three algorithms give the same performance, the joint algorithm reduces the CPU time by $16.5 \%$ compared to traditional interleaver algorithm. Similarly, the adaptive number of interleavers and SLM algorithm reduces the CPU time by $50.1 \%$. This shows the advantages of using this "adaptability" concept from processing power point of view.

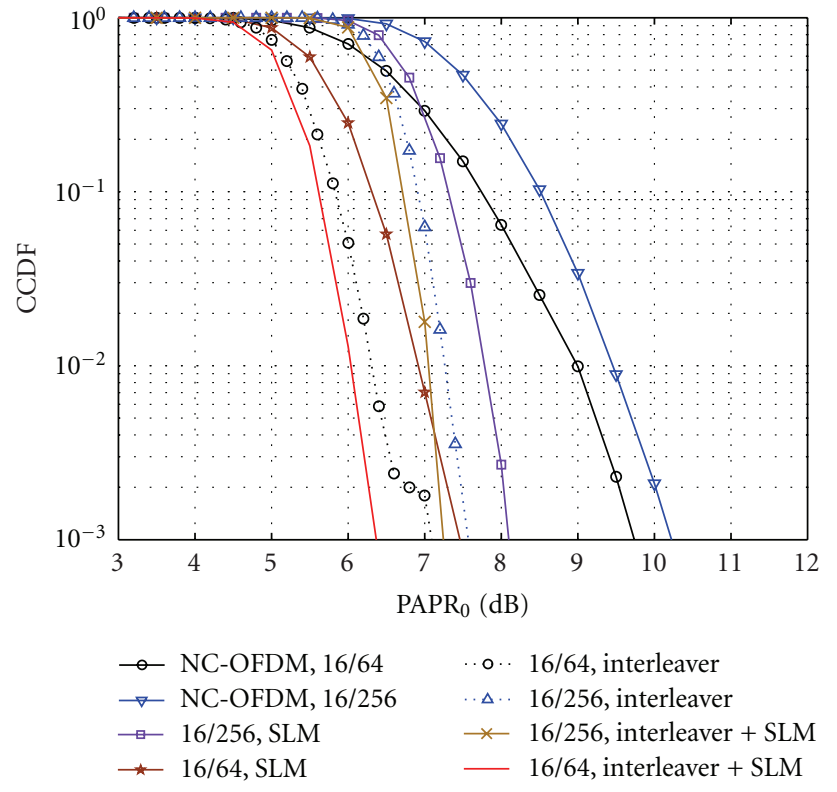

(a) QPSK

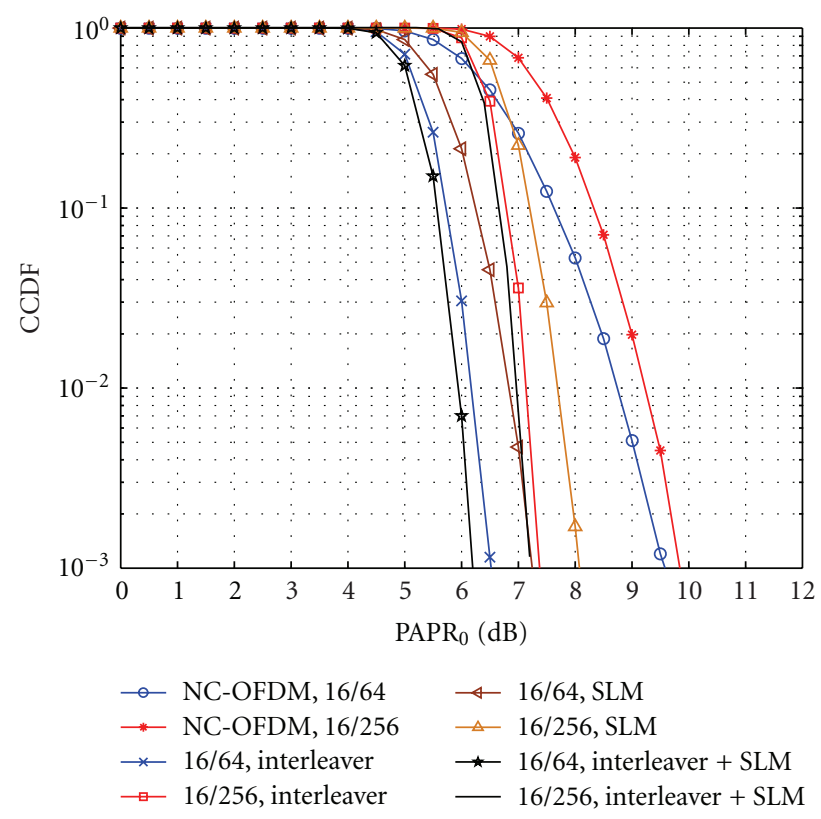

(b) $16 \mathrm{QAM}$

Figure 4: CCDF Performance for original, interleaving, SLM, and proposed joint algorithm systems.

\section{Conclusions}

In this paper, we have introduced three proposed algorithms for PAPR reduction of NC-OFDM signals. The first algorithm combines interleaving and SLM in a way that is suitable for NC-OFDM. With the proposed scheme, PAPR reduces by value around $3.0 \mathrm{~dB}$ compared to normal NCOFDM systems. Then we have proposed a low complexity technique which is adaptive number of interleavers, to reduce PAPR of NC-OFDM signals. The last algorithm applies the "adaptability" concept to the joint interleavers-SLM 


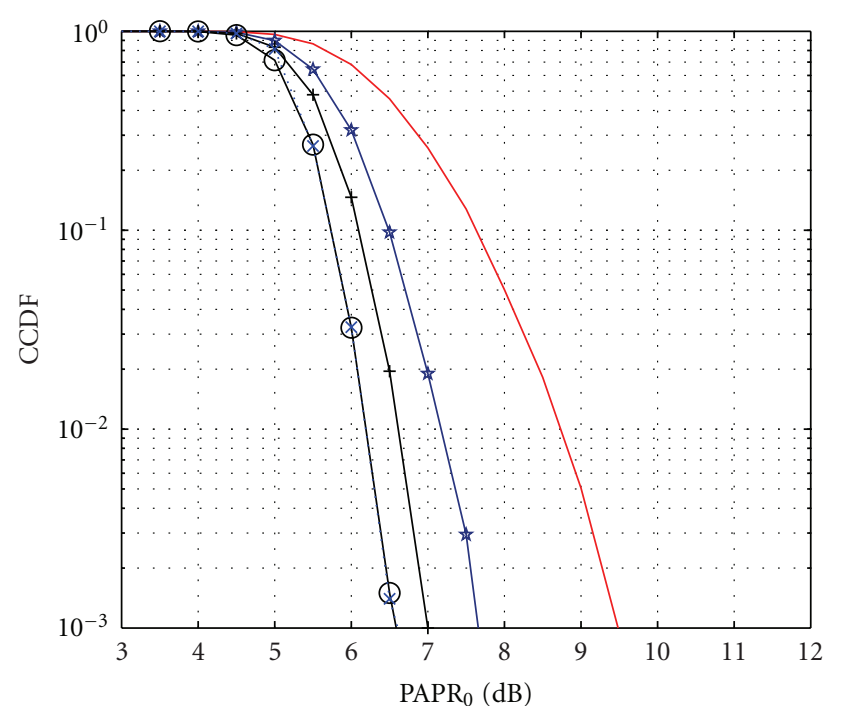

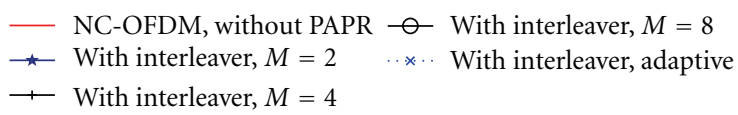

Figure 5: CCDF performance for 16QAM-modulated NCOFDM signals with different $M$ interleavers and proposed adaptive interleaver.

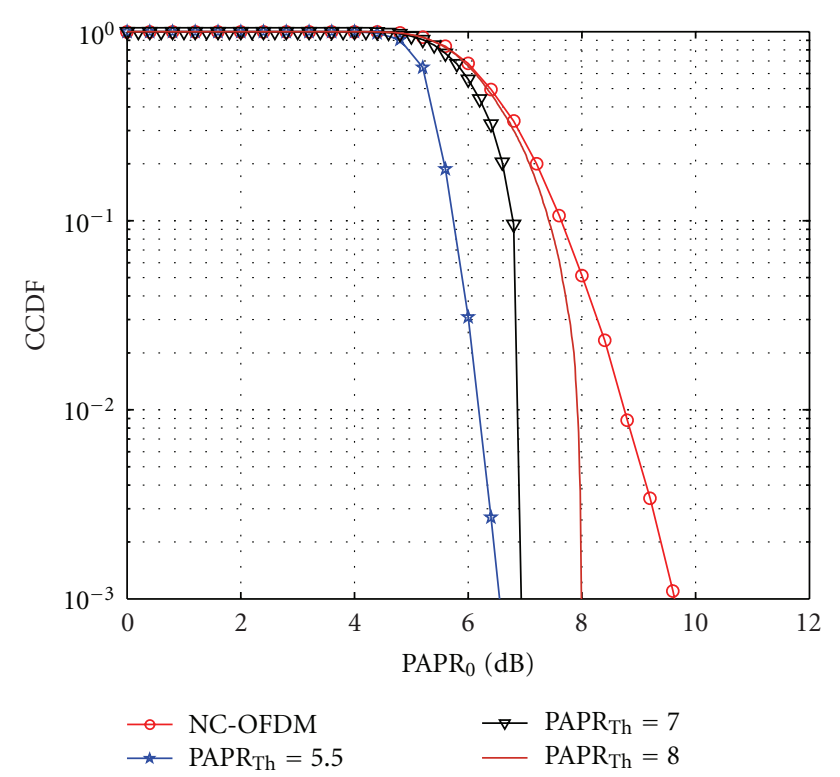

FIGURE 6: CCDF performance for 16QAM-modulated NC-OFDM signals for a proposed adaptive interleaver with different value of $\mathrm{PAPR}_{\mathrm{Th}}$.

technique. Simulation results showed the enhancements in PAPR and the processing power required using the adaptive algorithms, without loss in performance compared to the static algorithms.

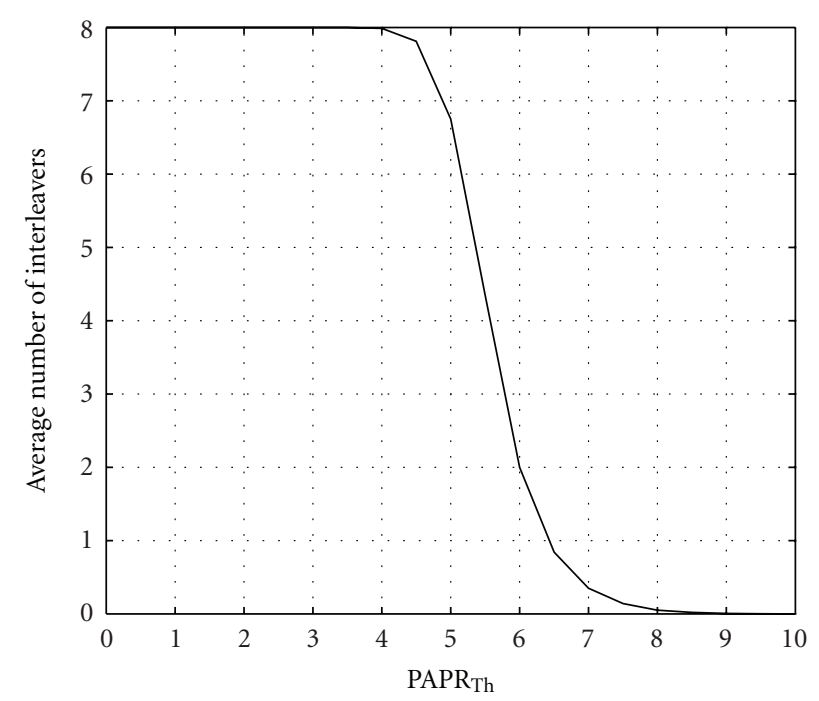

Figure 7: Average number of interleavers versus threshold $\mathrm{PAPR}_{\mathrm{Th}}$.

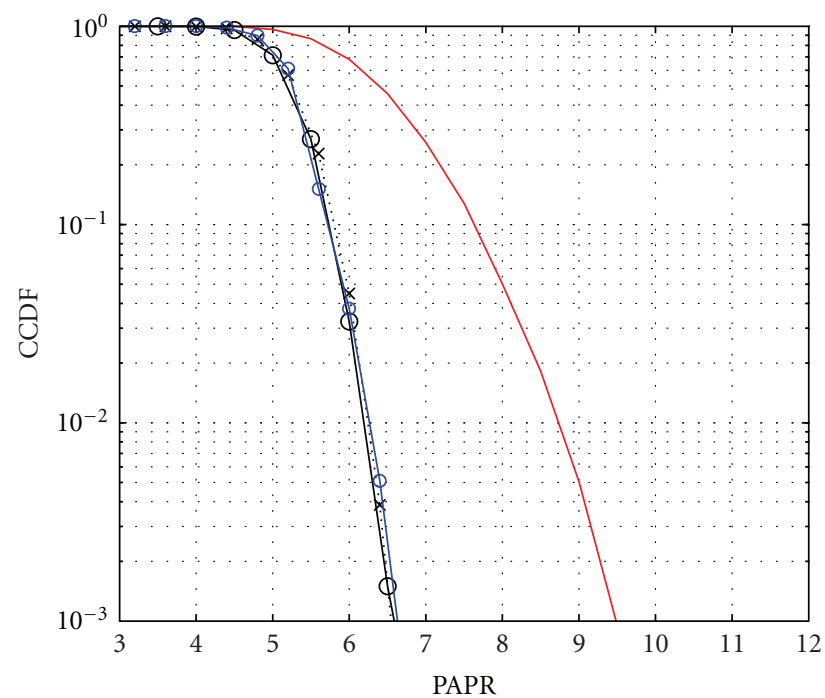

- NC-OFDM, without PAPR

- - With interleaver, $M=8$

..*. With $M=3$ interleaver $+D=4$ SLM

- With $M=5$ interleaver $+D=4$ SLM, adaptive

FIGURE 8: CCDF performance for 16QAM-modulated NC-OFDM signals with $M=8$ interleavers, proposed joint interleaver with SLM, and proposed adaptive joint algorithm.

\section{References}

[1] Federal Communications Commission, "Spectrum policy task force report," FCC Document ET Docket 02-155, 2002.

[2] J. Mitola, Cognitive radio: an integrated agent architecture for software defined radio [Ph.D. thesis], KTH, Stockholm, Sweden, 2000.

[3] S. Haykin, "Cognitive radio: brain-empowered wireless communications," IEEE Journal on Selected Areas in Communications, vol. 23, no. 2, pp. 201-220, 2005.

[4] S. Kumar, J. Sahay, G. K. Mishra, and S. Kumar, "Cognitive radio concept and challenges in dynamic spectrum access 
for the future generation wireless communication systems," Wireless Personal Communications, vol. 59, no. 3, pp. 525-535, 2011.

[5] H. Mahmoud, T. Yücek, and H. Arslan, "OFDM for cognitive radio: merits and challenges," IEEE Wireless Communications, vol. 16, no. 2, pp. 6-15, 2009.

[6] R. Rajbanshi, A. M. Wyglinski, and G. J. Minden, "Peak-toaverage power ratio analysis for NC-OFDM transmissions," in Proceedings of the IEEE 66th Vehicular Technology Conference (VTC'07), pp. 1351-1355, October 2007.

[7] A. Ghassemi, L. Lampe, A. Attar, and T. A. Gulliver, "Joint sidelobe and peak power reduction in OFDM-based cognitive radio," in Proceedings of the IEEE 72nd Vehicular Technology Conference Fall (VTC'10), September 2010.

[8] C. Zhang, "Cognitive non-continuous carrier interferometry orthogonal signal division multiplex transmission in autonomous vehicular communications," IEEE Journal on Selected Areas in Communications, vol. 29, no. 1, pp. 37-47, 2011.

[9] R. Rajbanshi, A. M. Wyglinski, and G. J. Minden, "Adaptivemode peak-to-average power ratio reduction algorithm for OFDM-based cognitive radio," in Proceedings of the IEEE 64th Vehicular Technology Conference (VTC '06), pp. 1350-1354, September 2006.

[10] L. Xin, W. Qihui, and Y. Yang, "A new SLM OFDM scheme with low complexity for PAPR reduction in CR system," in Proceedings of the IEEE International Conference on Wireless Information Technology and Systems (ICWITS '10), pp. 1-4, September 2010.

[11] T. Jiang and Y. Wu, "An overview: peak-to-average power ratio reduction techniques for OFDM signals," IEEE Transactions on Broadcasting, vol. 54, no. 2, pp. 257-268, 2008.

[12] O. A. Nasr and B. Daneshrad, "Circuit-energy aware discrete bit loading," in Proceedings of the IEEE International Conference on Communications (ICC '10), pp. 1-5, May 2010. 

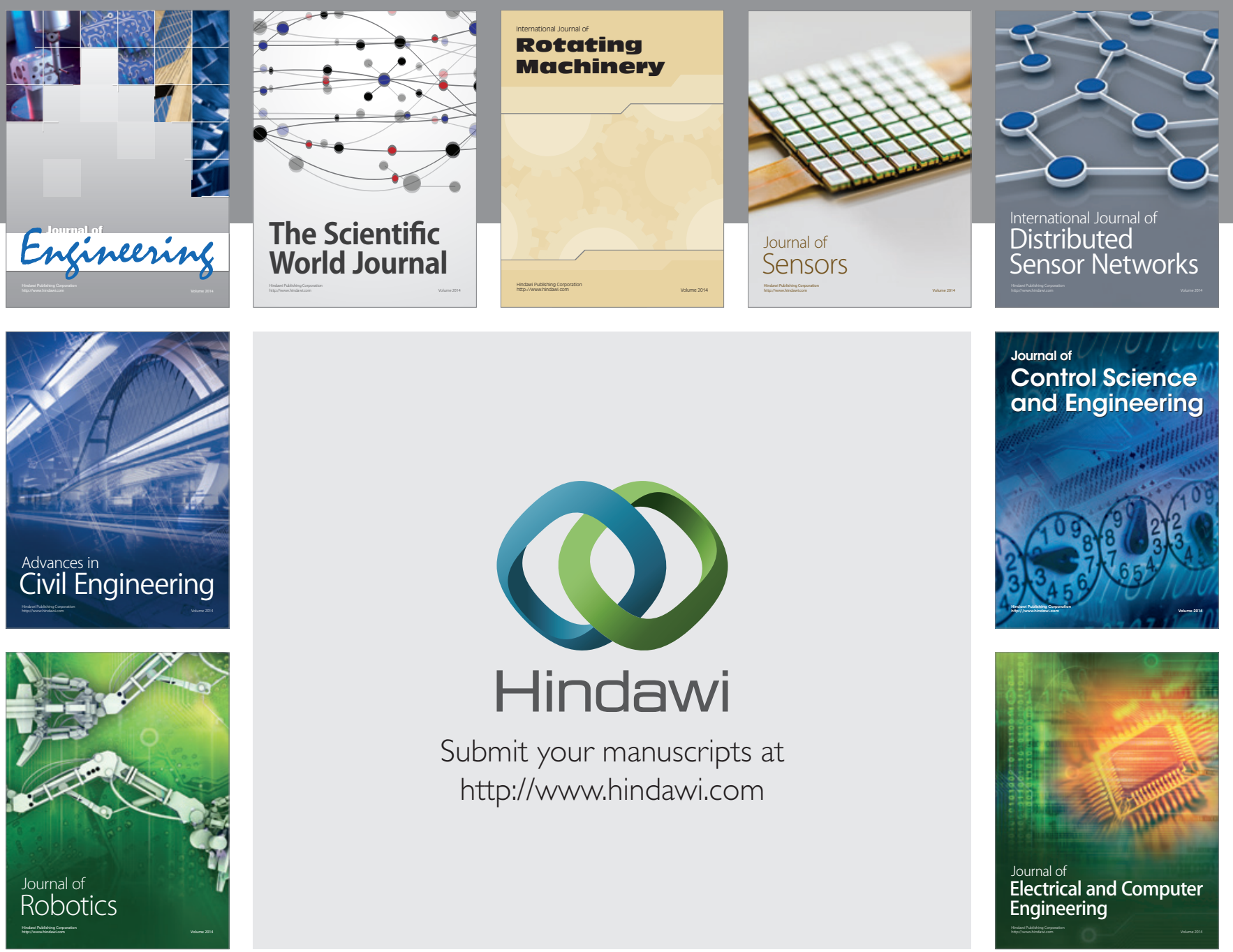

Submit your manuscripts at

http://www.hindawi.com
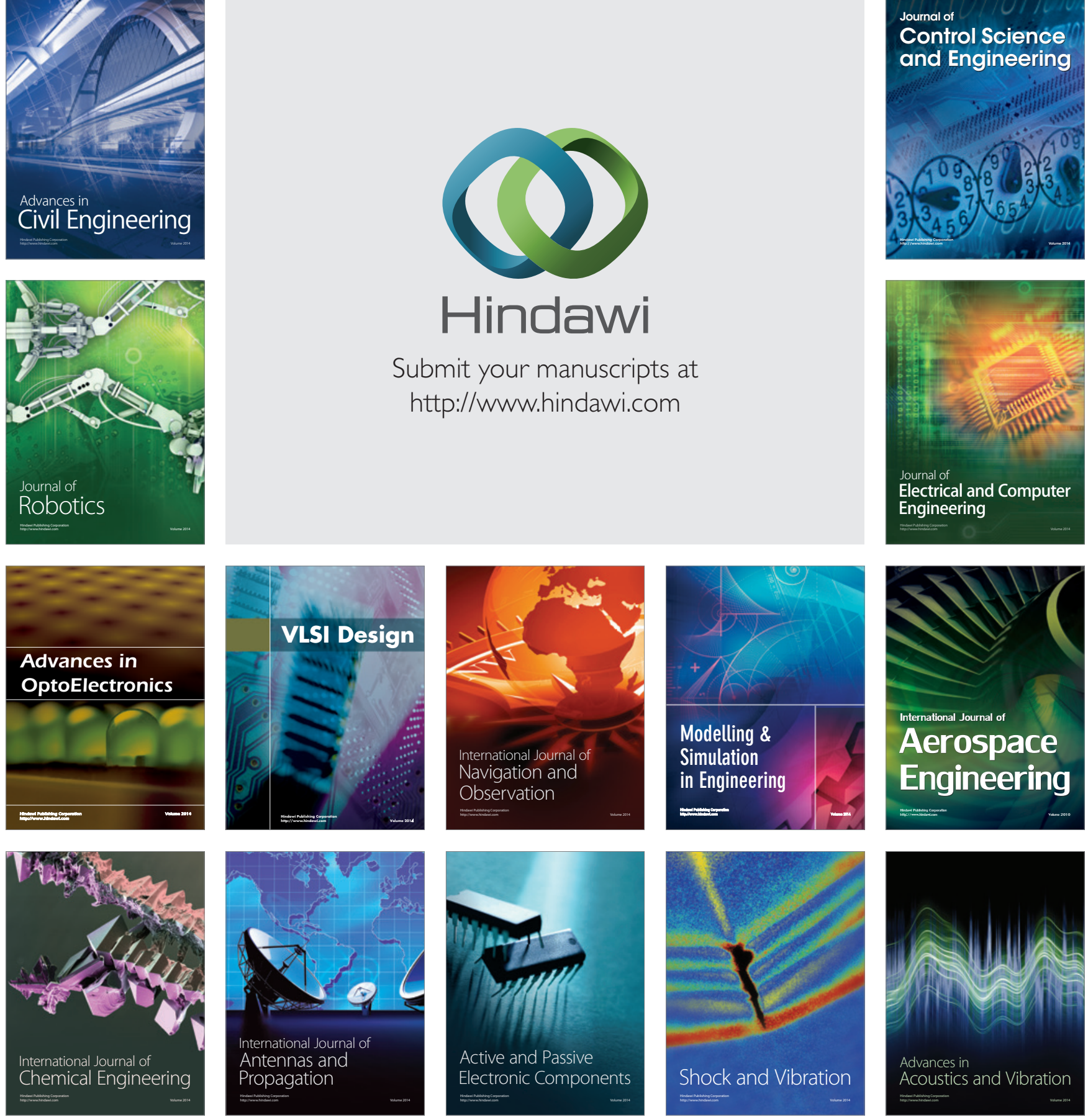